\section{Fisiopatología de la historia natural de la uropatía obstructiva secundaria a obstrucción de salida de la vejiga por hiperplasia benigna de próstata: implicaciones en la práctica clínica}

\author{
Pathophysiology of the natural history \\ of obstructive uropathy secondary to \\ bladder outlet obstruction resulting \\ from benign prostatic hyperplasia: \\ Implications in clinical practice
}

Antonio Alcántara Montero

\section{Sr. Editor:}

La uropatía obstructiva es una alteración común en la que un problema anatómico o funcional causa la obstrucción del flujo urinario normal. Esta obstrucción puede ocurrir en cualquier parte del tracto urinario. La obstrucción de salida de la vejiga, secundaria a la hiperplasia benigna de próstata, representa la causa más frecuente de uropatía obstructiva en pacientes adultos, afectando a $30 \%$ de los varones mayores de 60 años de edad. ${ }^{1}$

La historia natural de la uropatía obstructiva es similar a la de las cardiopatías. La vejiga, al igual que el corazón, es un órgano muscular hueco que recibe fluido y lo expulsa con fuerza. Además, reacciona ante un aumento de la carga de trabajo, pasando por fases sucesivas de compensación, descompensación (con posterior fracaso) o insuficiencia. ${ }^{2}$
Centro de Salud José María Álvarez, Don Benito (Badajoz, España). Miembro del Grupo de Trabajo de Urología (Nefrología y Vías Urinarias) de la Sociedad Española de Médicos de Atención Primaria (SEMERGEN)

Recibido: marzo 2017

Aceptado: agosto 2017

Correspondencia Antonio Alcántara Montero a.alcantara.montero@hotmail.com

DOI: https://doi.org/10.24245/revmexurol.v77i5.1519 
El motor primario que mueve el desarrollo de la uropatía obstructiva es la tensión mecánica. Durante la obstrucción de salida de la vejiga, las paredes de este órgano experimentan un aumento de tensión mecánica en el proceso de micción. Esta tensión activa las señales inducibles por estiramiento, lo que provoca modificaciones morfológicas y funcionales en el epitelio, las células del músculo liso, la matriz extracelular y la red neuronal. ${ }^{3}$

La hipertrofia de la pared de la vejiga representa el primer cambio anatómico que ocurre después de la obstrucción de salida de la vejiga, y está causada por la hipertrofia y proliferación de las células de músculo liso y la acumulación de colágeno, siendo ésta última la más importante de las tres., ${ }^{3,4}$ A medida que aumenta el grosor de la pared de la vejiga incrementa la obstrucción del detrusor. ${ }^{5-6}$ Adicionalmente, la hipertrofia muscular y la acumulación de colágeno disminuyen significativamente el flujo sanguíneo hacia el detrusor $y$, por tanto, se altera la difusión del oxígeno. A pesar de ello, durante la fase de compensación se estimula la angiogénesis, lo que provoca un aumento de la densidad vascular y el flujo sanguíneo en función del incremento de la masa vesical. ${ }^{7}$

El proceso desencadenado por la hipertrofia del detrusor se resume de la siguiente manera:2,5,8-9

- La hipertrofia del detrusor aumenta su poder de contractilidad para mantener un vaciado efectivo a pesar de la obstrucción.

- La vejiga genera una mayor presión para sobreponerse del aumento de la resistencia de salida, con una mínima o incluso inexistente orina postmiccional residual.

- La vejiga hipertrófica puede provocar presiones 2-4 veces más elevadas que los valores normales en un intento por forzar el paso de la orina a través de la obstrucción.
Es importante considerar que, en los estadios precoces de la obstrucción, la presión no se transmite a los uréteres ni a la pelvis renal, debido a la acción de la unión ureterovesical. En estos estadios la función de la vejiga permanece relativamente normal $y$, en general, los cambios limitados en las características del flujo que ocurren durante esta fase con función compensada, no suelen representar un motivo suficiente para que el paciente busque atención médica. ${ }^{7}$ Pero, ¿qué ocurre cuando la obstrucción persiste? El aumento de la presión vesical ejerce presión en la mucosa localizada entre los haces de los músculos superficiales, causando la formación de pequeños bultos. En modelos experimentales se ha evidenciado la acumulación progresiva de colágeno entre las fibras musculares. Las consecuencias de la acumulación de colágeno son bien conocidas; la fibrosis que se genera estimula la expresión de factores angiostáticos que inhiben la angiogénesis y disminuyen el flujo sanguíneo, para de esta forma originar el daño isquémico. ${ }^{10}$ La isquemia del detrusor causa múltiples alteraciones responsables de la progresión gradual y con ello descompensación de la vejiga, por ejemplo: alteraciones mitocondriales, estrés oxidativo, aumento del metabolismo anaeróbico, acumulación de glucógeno, sobrecarga de calcio y activación de enzimas de degradación, y denervación de la vejiga (Figura 1). ${ }^{711}$ Como consecuencia, las manifestaciones clínicas por descompensación de la vejiga incluyen: reducción de la contractilidad del detrusor, disfunción de la vejiga (la más importante) y aumento de la orina residual postmiccional. ${ }^{7,12}$

Cuando la obstrucción persiste, la descompensación provoca insuficiencia. ${ }^{2}$ En el estadio terminal de la uropatía obstructiva la vejiga se caracteriza por tener una estructura fibrosa, capacidad disminuida, disfunción y contractilidad deficientes. ${ }^{13}$ En casos extremos las consecuencias de esta alteración resultan en descompensación del tracto urinario superior. 


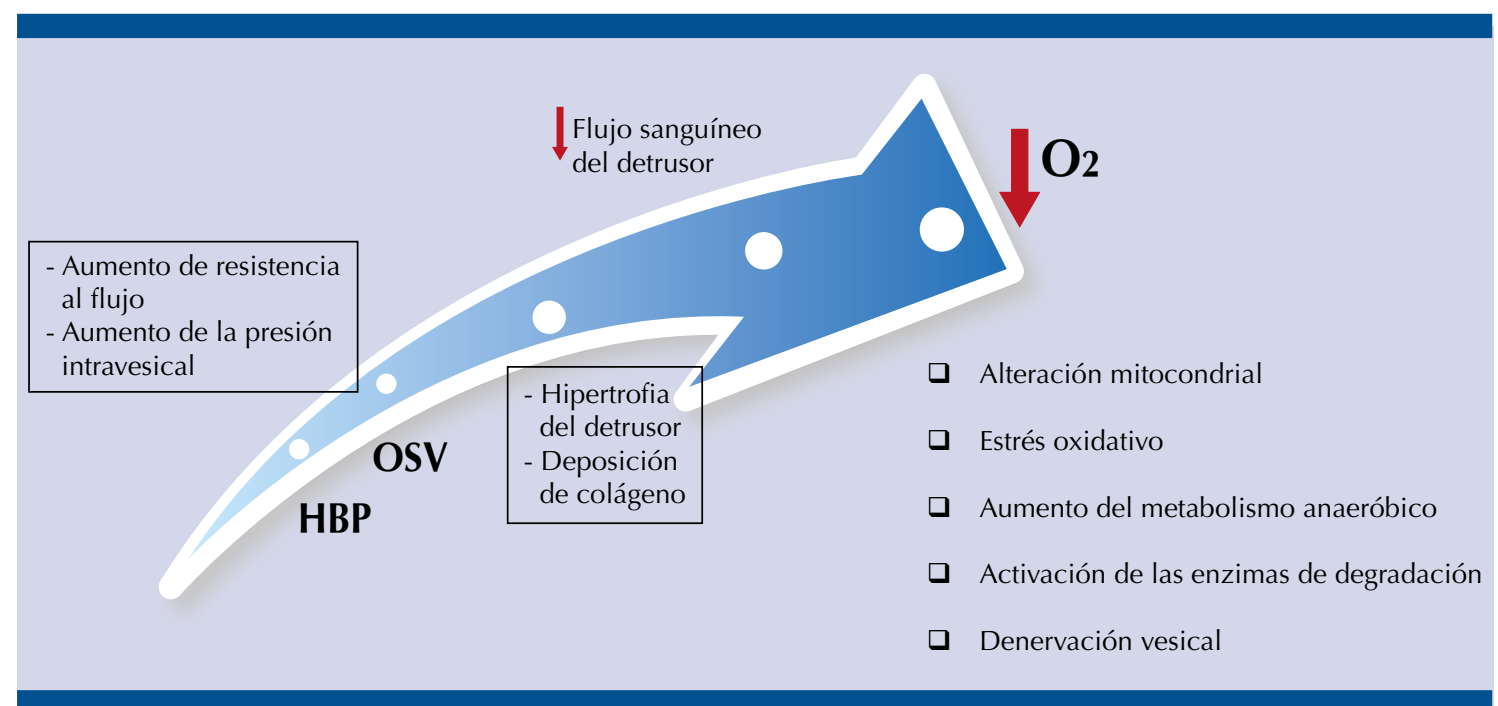

Figura 1. Historia natural de la uropatía obstructiva secundaria a obstrucción de salida de la vejiga por hiperplasia benigna de próstata (Fuente: Komninos C, et al.7).

HBP: hiperplasia benigna de próstata; OSV: obstrucción de salida de la vejiga.

Pero, ¿es posible cambiar o revertir la historia natural de la uropatía obstructiva? Existe evidencia limitada, procedente principalmente de modelos animales, que sugiere que la historia natural del trastorno puede interrumpirse o revertirse. Por otro lado, se ha demostrado que el uso de alfa1-bloqueantes reduce significativamente $(13 \%)$ el peso vesical, estimado por ecografía después de 6 meses de tratamiento en pacientes con hiperplasia benigna de próstata. Sin embargo, se requieren estudios adicionales y más extensos para confirmar un posible efecto terapéutico de los alfa1-bloqueantes sobre el músculo detrusor. ${ }^{14}$

En conclusión, las estrategias encaminadas a disminuir la presión intravesical podrían representar una forma válida de interrumpir o revertir la historia natural de la uropatía obstructiva secundaria a la obstrucción de salida de la próstata. Además, intervenciones precoces pueden proporcionar mayores oportunidades para recuperar la función vesical.

\section{REFERENCIAS}

1. Tseng TY, Stoller ML. Obstructive uropathy. Clin Geriatr Med. 2009;25:437-43.

2. Elmissiry MM, Ali AG, Abulfotooh A, Moussa AA, Ali GA. Factors determining the amount of residual urine in men with bladder outlet obstruction: Could it be a predictor for bladder contractility? Arab J Urol. 2014;12:214-8.

3. Mirone V, Imbimbo C, Longo N, Fusco F. The detrusor muscle: an innocent victim of bladder outlet obstruction. Eur Urol. 2007;51:57-66.

4. Duan LJ, Qi J, Kong XJ, Huang T, Qian XQ, Xu D, et al. MiR133 modulates TGF- $\beta 1$-induced bladder smooth muscle cell hypertrophic and fibrotic response: implication for a role of microRNA in bladder wall remodeling caused by bladder outlet obstruction. Cell Signal. 2015;27:215-27.

5. Tubaro A, De Nunzio C, Trucchi A, Palleschi G, Miano L. The effect of bladder outlet obstruction treatment on ultrasound-determined bladder wall thickness. Rev Urol. 2005;7(Suppl 6):S35-42.

6. Oelke $M$, Höfner K, Wiese B, Grünewald V, Jonas U. Increase in detrusor wall thickness indicates bladder outlet obstruction (BOO) in men. World J Urol. 2002;19:443-52.

7. Komninos $C$, Mitsogiannis I. Obstruction-induced alterations within the urinary bladder and their role in the pathophysiology of lower urinary tract symptomatology. Can Urol Assoc J. 2014;8:E524-30. 
8. Stein R, Gong C, Hutcheson J, Krasnopolsky L, Canning DA, Carr $\mathrm{M}$, et al. The fate of urinary bladder smooth muscle after outlet obstruction--a role for the sarcoplasmic reticulum. Adv Exp Med Biol. 2003;539(Pt B):773-90.

9. Sakai T, Kasahara K, Tomita K, Ikegaki I, Kuriyama H. 5-Hydroxytryptamine-induced bladder hyperactivity via the 5-HT2A receptor in partial bladder outlet obstruction in rats. Am J Physiol Renal Physiol. 2013;304:F1020-7.

10. Metcalfe PD, Wang J, Jiao $H$, Huang $\mathrm{Y}$, Hori $\mathrm{K}$, Moore RB, et al. Bladder outlet obstruction: progression from inflammation to fibrosis. BJU Int. 2010;106:1686-94.

11. Levin RM, Schuler C, Leggett RE, Callaghan C, Maknuru S. Partial outlet obstruction in rabbits: duration versus severity. Int J Urol. 2013;20:107-14.
12. Choi BH, Jin LH, Kim KH, Kang SA, Kang JH, Yoon SM, et al. Cystometric parameters and the activity of signaling proteins in association with the compensation or decompensation of bladder function in an animal experimental model of partial bladder outlet obstruction. Int J Mol Med. 2013;32:1435-41.

13. Blatt AH, Brammah S, Tse V, Chan L. Transurethral prostate resection in patients with hypocontractile detrusor--what is the predictive value of ultrastructural detrusor changes? J Urol. 2012;188:2294-9.

14. Sironi D, Levorato CA, Deiana G, Borgonovo G, Belussi $D$, Ranieri $A$, et al. Decrease of ultrasound estimated bladder weight during tamsulosin treatment in patients with benign prostatic enlargement. Arch Ital Urol Androl. 2002;74: 90-4.

\section{AVISO PARA LOS AUTORES}

Revista Mexicana de Urología tiene una nueva plataforma de gestión para envío de artículos: https://www.revisionporpares.com/index.php/RMUrol ahí podrá inscribirse a la base de datos administrada por el sistema Open Journal System (OJS) que ofrece las siguientes ventajas para los autores:

- Subir sus artículos directamente al sistema.

- Conocer, en cualquier momento, el estado de los artículos enviados, es decir, si ya fueron asignados a un revisor, aceptados con o sin cambios, o rechazados.

- Participar en el proceso editorial corrigiendo y modificando sus artículos hasta su aceptación final. 